\begin{tabular}{|c|l|}
\hline Title & 3D topology optimization using an immune algorithm \\
\hline Author(s) & Campelo, Felipe; Watanabe, Kota; Igarashi, Hajime \\
\hline Citation & $\begin{array}{l}\text { COMPEL : The International Journal for Computation and Mathematics in Electrical and Electronic Engineering, 26(3), } \\
\text { https://doi.org/10.1108/03321640710751145 }\end{array}$ \\
\hline Issue Date & 2007 \\
\hline Doc URL & http://hdl.handle.net/2115/28235 \\
\hline Type & article (author version) \\
\hline File Information & CIJCM26-3.pdf \\
\hline
\end{tabular}

Instructions for use 


\section{D Topology Optimization Using an Immune Algorithm}

Felipe Campelo, Kota Watanabe, and Hajime Igarashi

Laboratory of Hybrid Systems

Graduate School of Information Science and Technology, Hokkaido University

Kita 14, Nishi 9, Kita-ku, Sapporo 060-0814, JAPAN

FAX : +81 11-706-7670

pinto@em-si.eng.hokudai.ac.jp; \{watanabe,igarashi\}@ssi.ist.hokudai.ac.jp 


\title{
3D Topology Optimization Using an Immune Algorithm
}

\begin{abstract}
Purpose - The paper introduces an evolutionary algorithm based on the artificial immune systems paradigm for topology optimization in 3D.

Design/methodology/approach -The 3D topology optimization algorithm is described, and experimentally validated on an electromagnetic design problem.

Findings - The proposed method is capable of finding an optimal configuration for the validation problem used.

Research limitations/implications - More tests are needed in order to fully assert the capabilities of the algorithm. Moreover, further improvements are needed in order to obtain smoother topologies at the end of the optimization procedure.

Practical implications - The paper presents a novel tool for the design of electromagnetic devices, using a topology optimization approach.

Originality/value - So far most of the topology optimization algorithms did not tackle true $3 \mathrm{D}$ problems, and the ones capable of $3 \mathrm{D}$ optimization do it at high computational costs. The algorithm presented here is capable of true 3D design at a reasonable computational budget.
\end{abstract}

Keywords - topology optimization, electromagnetic design, immune algorithms

Paper type - Research paper 


\section{Introduction}

Shape optimization of engineering devices usually means maximizing a given performance measure by varying the dimensions of a basic shape set by the designer, in a process sometimes called parametric optimization. While this strategy can lead to very good results, it is fundamentally dependent on the basic configuration provided, i.e., the optimization algorithm is not able to introduce fundamental changes in the topology of the device being designed. In other words, the algorithms lack the ability to develop innovative solutions.

A different approach to the geometrical design of such devices is the Topology Optimization (TO) paradigm. Instead of being limited to predefined shapes and variables, in TO the material properties at every point of the design space are considered in the design processes. By allowing the free variation of the material properties at any point of the design space, TO algorithms are able to return novel or unexpected solutions, independently from any initial design (Campelo, 2006).

The computational cost associated with TO methods, however, remains as an obstacle to a broader use of this kind of technique. This has somehow limited the use of TO algorithms to $2 \mathrm{D}$ problems or problems with axial symmetry or planar structure, where the design space can be reduced to two dimensions. Indeed, there are very few works in the literature dealing with 3D design of electromagnetic devices using TO (Yoo, 2000; Yoo, 2002; Okamoto, 2006).

Among the methods used for two-dimensional TO, the Clonal Selection Algorithm for Topology Optimization (TopCSA) (Campelo, 2006) presents some interesting features. This technique is an evolutionary algorithm that presents local and global search 
characteristics at a reasonably low computational cost (Campelo, 2006; Watanabe, 2006), valuable features when designing electromagnetic devices.

In this work we extend the TopCSA to deal with real 3D problems. The operators of the algorithm are adapted, and the technique is tested on the design of a magnetic device. The paper is organized as follows: Section II presents a brief overview on methods used for topology optimization; the 3D version of the TopCSA is presented in Section III; in Section IV the multigrid method, used by the TopCSA as the numerical solver, is described; the results obtained by the proposed technique for the design of a perpendicular magnetic recording head are presented in Section V; final remarks, conclusions and ideas for future work close the paper in Section VI.

\section{Topology Optimization}

As explained in the previous section, topology optimization algorithms search for optimum distributions of material within a finite design space. In order to implement algorithms for this task, appropriate representations of the candidate solutions are essential. In this section, we present some of the most popular representation techniques found in the literature.

\section{Homogenization Design Method}

The Homogenization Design Method (HDM) for topology optimization was first introduced by Bendsøe and Kikuchi (Bendsøe, 1988), for applications in structural design. In the HDM, the design space is divided in a number of perforated cells, and the macroscale optimal shape is determined by the optimization of the size of these micro- 
holes in the structure. At the end of the optimization process, elements with large cavities will represent empty space, while elements with small cavities will represent filled regions, and therefore will form the structure of the solution. This method has been used in a number of works in electromagnetic design (Nishiwaki, 1998; Silva, 2000), and has the advantage of avoiding the complications of a discrete optimization approach, by transforming the problem to the continuous space (optimization of cavity sizes and orientations). The main drawbacks of this representation are the large number of optimization variables generated by this technique, for modeling the size and orientation of the cavities; the computational effort required for the calculation of the material constants for each element, which can be considerably high; and the need for the user to define thresholds for the intermediate elements to be considered as filled or as empty.

In 3D topology optimization, the HDM has been used in some works (Yoo, 2000; Yoo, 2002), coupled with the Sequential Linear Programming (SLP) optimization technique. However, a three dimensional model of the HDM's microvoid structure increases the number of design variables even more, from 3 per cell in the 2D case to 6 per cell, as seen in Figure 1.

\section{Optimized Material Distribution}

In order to overcome some of the negative points of the HDM, Rozvany (1992) proposed the Solid, Isotropic Microstructure with Penalty (SIMP) approach. It became later generally known as the Density Method (DM) or Optimized Material Distribution (OMD), depending on the field of application, and has been widely used in electromagnetic design (Byun, 1999; Mlejnek, 1993; Wang, 2002; Dick, 1996). One of 
the objectives of the OMD technique was to avoid the checkerboard-like structures generated by the use of the HDM, as well as to simplify the analysis and optimization processes in the design of the devices.

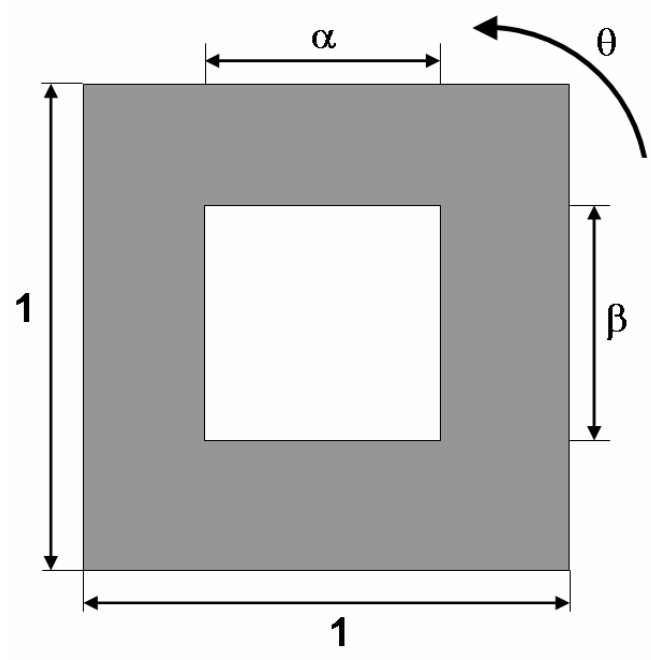

(a)

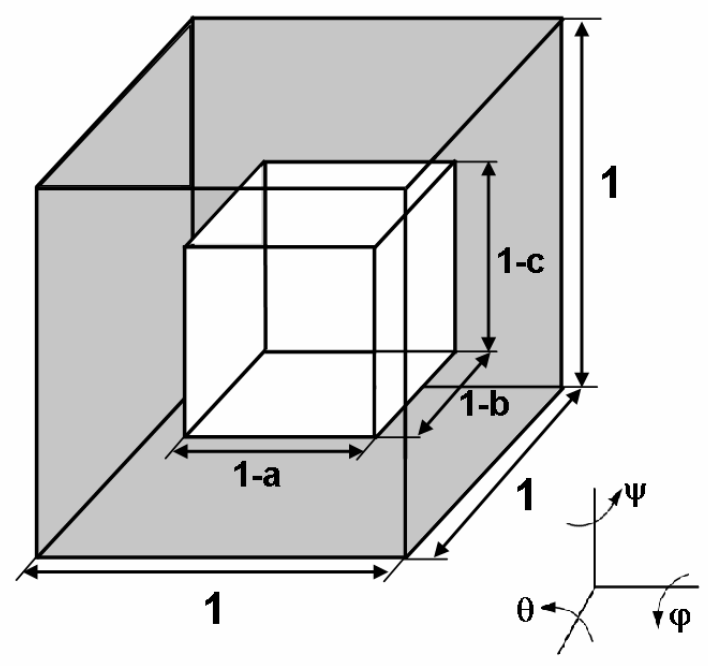

(b)

Figure 1: Microstructure in the HDM representation. In the 2D case (a), each element presents 3 variable dimensions, representing the size and angle of the hole; in 3D (b), this number increases to 6 , doubling the dimension and greatly increasing the complexity of the problem to be solved by the optimization algorithm.(Figures adapted from Nishiwaki (1998) and Yoo (2002), respectively).

Instead of considering each element as a region of material with a cavity, the OMD approach considers the normalized density of material in each element, varying from " 0 " (empty) to "1" (filled), as the main optimization variables. With the material properties being considered homogeneous within a given element, a great reduction in the number of optimization variables (from three to one per element in 2D problems, for instance) could be achieved. 
In the OMD, intermediate densities are penalized in order to make it unlikely for the final solutions to exhibit "gray" (i.e., intermediate) materials. The maintenance of intermediate densities during the optimization process is used for avoiding the need of discrete optimization techniques, and the relationship between the properties of "gray" materials and the normalized density is given by simple relations (Bendsøe, 1999).

In 2004, a modified version of the OMD, also based on ideas of the HDM, was proposed for the optimization of 3D structures (Yoo, 2004). The technique was also coupled with SQL for the solution of the optimization problem, and tested on the design of a C-core magnet. Comparisons between this modified OMD and the HDM show approximately the same performance and same computational cost for both approaches. The modified OMD can, however, exhibit a much higher computational cost, depending on the choice of the parameter related to the penalization of intermediate densities.

There is a general trend for works using OMD or HDM to use sensitivity-based optimization techniques for TO, in particular SLP or steepest-descent methods. In some cases of specific design applications, the derivative information can be obtained analytically and used in the optimization process. While this approach is computationally effective, since it require no additional function evaluations, it is very limited and cannot be extended to general cases. On the other hand, the numerical evaluation of derivatives is in most cases prohibitively expensive, from a computational point of view. Moreover, gradient-based optimization methods lack global exploration features, and may be trapped at local optima in multimodal problems. 


\section{Voronoi Diagrams}

The Voronoi representation was a first attempt towards unstructured representations for TO (Hamda, 2002). By unstructured it means a representation whose complexity is not dependent on a fixed discretization, an interesting characteristic that can allow finer meshes to be used in the solver without increasing the size of the candidate solutions' representation.

For describing the Voronoi representation, we must first define a Voronoi diagram (Hamda, 2002): consider a finite number of points, called Voronoi sites, contained in a given finite set within the $\mathbf{R}^{\mathbf{n}}$ space. Each Voronoi site $V_{i}$ defines a Voronoi cell, composed by all points in the design domain for which it is the closest site. A Voronoi diagram is the partition of the design domain defined by all the Voronoi cells. Figure 2 illustrates this concept better.

In a number of works published by Hamda (2000; 2002), Voronoi-based representations with a variable number of sites were used in conjunction with a specially adapted genetic algorithm for the optimization of mechanical structures in 2D and 3D. The main advantage of this representation is the independence of the inner representation of the candidate solutions (i.e., the representation used by the optimization algorithm) from the mesh used for the evaluation of the solution's performance. There are, however, some issues concerning the design of efficient operators for the evolution of the solutions.

\section{Binary Representations}

One of the most natural ways of encoding a candidate solution for a topology optimization problem is to represent it directly as a matrix of elements representing the 
"state" at each point of the design space. This approach, however, requires optimization methods capable of dealing with discrete problems. Due to this fact, it has been avoided in many works in the literature, in favor of continuous, real coded approaches (e.g., HDM or OMD).

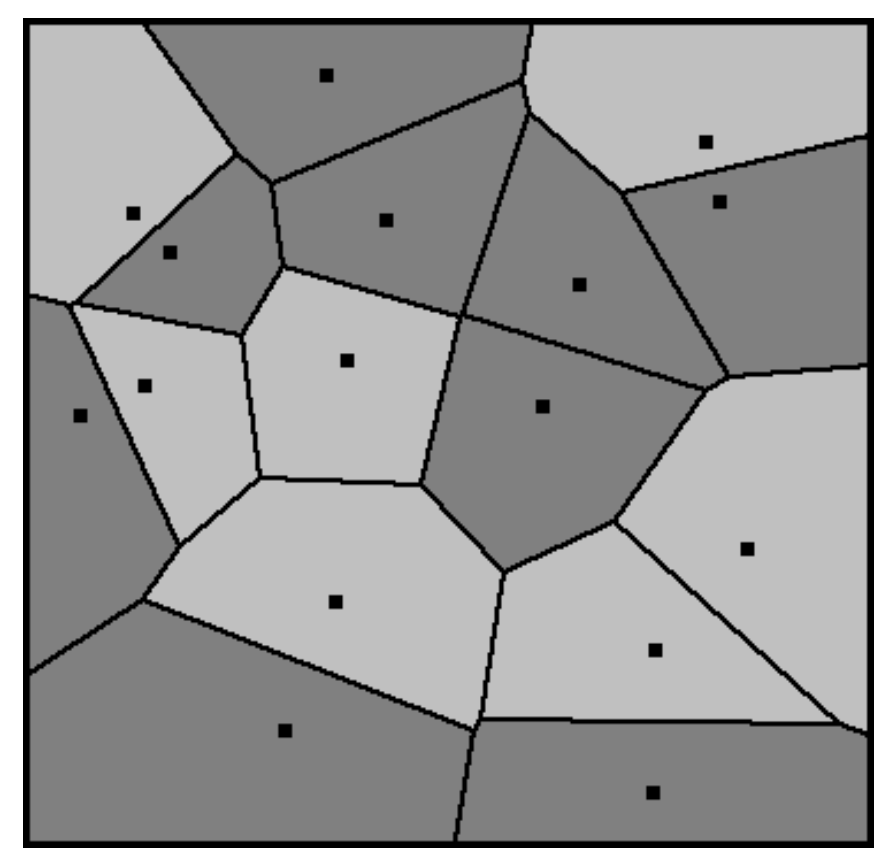

Figure 2: A 2D Voronoi diagram. The dots represent the Voronoi sites, and the lines show the partition of the design space in Voronoi cells. Light and dark gray regions could represent different materials in a topology optimization process. (Example generated using Voroglide (Icking, 2001))

In 1993, a direct approach to the discrete problem, using binary representation and a discrete optimization method (Genetic Algorithm) was proposed (Chapman, 1993). The design space was discretized, with all the elements forming a binary, one dimensional array of "zeros" and "ones" (meaning empty and filled elements, respectively). By working directly with the existence/nonexistence of material in the elements, this methodology does not require the consideration of gray materials, and therefore simplify 
the optimization process (Chapman, 1993; Jakiela, 2000). Binary representations were later expanded, by considering a two-dimensional, direct representation of the design space (Kane, 1996), in order to allow a more effective use of Genetic Algorithms over TO problems in $2 \mathrm{D}$.

Most of the works in literature employing binary or Voronoi representations of the design space make use of Genetic Algorithms for evolving optimal topologies, since GAs are able to work more easily with binary variables (or variable-length lists of points, in the case of Voronoi representations). Specific genetic operators can be developed for the 2D or 3D encoding of the search space (Im, 2003), in order to improve the convergence characteristics of the GA. Given the global search capabilities provided by the genetic operators, it is possible for the algorithm to locate global optimal distributions of material.

There are, however, some issues that limit the use of GA-based methods in topology optimization. The first is the high computational cost associated with the evolution of a large population of candidate solutions. While smaller populations can be used, in most cases it will imply in premature convergence of the algorithm due to the small genetic diversity and the genetic drift introduced by crossover operators. Second, since the GA's lack local exploration features, it takes a long time to evolve from a solution lying in the vicinity of a given optimum to the actual optimal point. Finally, there is the problem in designing efficient operators, e.g., Crossover.

An interesting study on various strategies of shape representation for evolutionary topology optimization, including binary representations and others not discussed here (e.g., H-representations), can be found in Schoenauer's work (Schoenauer, 1996). 


\section{The Clonal Selection Algorithm for Topology Optimization}

In a previous work (Watanabe, 2006), the authors propose a new technique for the optimization of $2 \mathrm{D}$ topologies. The TopCSA is an evolutionary algorithm based on the Clonal Selection Principle (de Castro, 2002), and presents both global and local search characteristics at an acceptable computational cost. In the same paper, the algorithm is employed for the optimization of an MRI coil and a perpendicular magnetic recording head. The results obtained for those two problems show that the algorithm was able to effectively discover novel configurations of material as solutions for both problems. In another work (Campelo, 2006), the algorithm was tested in the design of another MRI device, also showing promising results. In both cases, however, the problems were somehow simplified by the fact that the problems presented linear symmetry, i.e., only the 2D model of an actual 3D device was designed.

In this section, we describe an adapted version of the TopCSA for use in true 3D design optimization. For the sake of brevity, some details of the original algorithm will be skipped. A more detailed description can be found in the literature (Campelo, 2006).

In this $3 \mathrm{D}$ version, the TopCSA represents each candidate solution as a three dimensional binary matrix: the search space is divided in a number of hexahedral elements, corresponding, for example, to elements in a finite-element mesh. Each element has associated to it a single binary value, corresponding to either the presence or absence of material in that particular region of the space. The representation of the design space is, in all aspects, similar to the one used by Okamoto (2006), where a sensitivitybased technique is used for optimization. 
The algorithm starts by generating a population $\boldsymbol{A} \boldsymbol{b}$ composed of $N_{P o p}$ random initial distributions of material in the design space. The iterative cycle then starts, with the evaluation of each candidate solution $A b_{i}$ over a given performance function. The candidate solutions are sorted according to their performance, and the $N_{S e l}$ best ones are selected for the Clonal Expansion process: for a number of times proportional to their position in the performance ranking (calculated by:

$$
N_{C}^{i}=\frac{\beta N_{P o p}}{i}
$$

where $i$ is the position in the ranking and $\beta$ is a user-defined parameter), an exact copy (clone) of the antibody is generated, and subject to either the Maturation process or to one of the Global Operators, as explained below. After that, the clone is evaluated, and replaces $A b_{i}$ if it presents better performance.

After the Clonal Expansion process, the solutions in $\boldsymbol{A b}$ that were not selected for cloning are replaced by new, randomly generated configurations of material, a process called Diversity Generation. The iterative cycle is repeated until some user-defined stop criterion is met.

\section{Maturation}

The Maturation operator acts on the "surface" of the material, i.e., on elements that are set as filled and present at least one interface with an empty element. As in Okamoto's works (Okamoto, 2006), the TopCSA uses a 3D von Newmann neighborhood rule (Weisstein, 2003), where only the elements that share faces are considered to be neighbors to each other. 
The Maturation process is implemented as follows: a given number of surface elements is chosen randomly, and then mutated, according to the following procedure:

- Determine the interface side of the element. If the element presents more than one interface side, select one randomly;

- Randomly determine the mutation direction $( \pm 1)$;

- If the direction is positive, the neighboring empty element is converted to filled; else, the element itself is converted to empty.

This operator present some specific characteristics: first, it is a local operator, since it acts only on the immediate neighborhood of the affected elements. Second, the advance or retreat of the material boundary happens only perpendicularly to the interface side.

\section{Global Operators}

The TopCSA presents three Global Operators: Cleaning, Surface Smoothing, and Macromutation. These operators are exclusive both mutually and with the Maturation operator, i.e., a clone can be subject to only one of them during a given cycle of the Clonal Expansion. The name Global comes from the fact that these operators do not act on one individual (local) element of the material distribution, but instead on all elements of the matrix that present certain characteristics characteristic, as explained below:

Cleaning: checks the neighborhood of all elements from the clone's distribution of material, and inverts the state of elements that present material interfaces at all six sides. In other words, it is responsible for removing individual isolated filled elements in the middle of empty regions, and vice versa. 
Surface Smoothing: acts similarly to the Cleaning, but instead of acting in elements completely surrounded by different ones, it operates on both sides of the material boundary, checking for elements that present five boundary faces (individual protruding filled elements or intruding empty elements). Both the Cleaning and the Surface Smoothing operators act quite similarly to the Topology Smoother (Okamoto2006).

Macromutation: consists in defining a random-sized, random-placed polyhedral region in the clone's distribution of material and setting all elements in the region to the same state (either filled or empty), randomly chosen. This operator helps the algorithm exploring new regions of the search space, e.g., evolving shapes with holes in the middle of material regions.

\section{Matrix Refinements}

In order to accelerate the convergence of the TopCSA, the coarseness of the material distribution is varied along the optimization process. The designer defines the desired initial and final dimensions of the matrix representing the search space, as well as a stabilization threshold for the refinement of the matrix (e.g., a given number of objective function evaluations without gains in the performance of the best solution). The algorithm starts with a coarser mesh in the first generations, in order to identify the general location of material in the search space; after that, it refines the mesh every time the stabilization threshold is reached, until the final resolution is achieved. After this point, the algorithm proceeds without further refinements, until the stop criteria are satisfied. 


\section{Enforcing Smoothness}

In the topology optimization of engineering devices, one is usually concerned about the smoothness of the solutions. Too rough, irregular, or perforated topologies may be too expensive or even unfeasible from an implementation point of view. Conversely, smoother, more homogeneous shapes are preferred, even if they lead to slightly worse performance of the device under consideration.

In order to force the TopCSA to evolve smoother shapes, a simple strategy is employed: since the algorithm already possesses operators for smoothing the clones, we define an allowance $\left(A_{s} \in[0,1]\right)$ for the performance of clones subject to one of these operators. A smoothed clone is then allowed to replace the "parent antibody" if its affinity value is higher than $\left(1-A_{s}\right)$ times the affinity of the "parent". With this simple modification it is possible to keep an evolutionary pressure for the algorithm in the direction of more regular-shaped topologies.

\section{The Multigrid Method}

It is known that linear solvers such as Gauss-Seidel methods tend to eliminate the highfrequency components of the residue in linear equations given by the finite element analysis more rapidly than the low-frequency components. The multi-grid method (Watanabe, 2003) is based on this property, that is, the high-frequency residual components are eliminated on a fine mesh in a small number of iterations of the linear solver. The remaining residual components are then projected onto a coarser mesh, in which they now are considered as high frequency components that can again be eliminated in a small number of iterations. 
The multigrid method solves the given equations by successively performing this process. The geometric multi-grid method used in this work requires hierarchical meshes with different densities. In this study, the finer meshes are obtained by dividing each coarse element into 4 (in the $2 \mathrm{D}$ case) or 8 (3D case) finer elements using the middle point of each edge. Although there are many variations of the multigrid method, all these variations are based on the coarse grid correction. The procedure of the two-grid V-cycle method that is used in conjunction with the TopCSA for the electromagnetic analysis is shown in Figure 3.

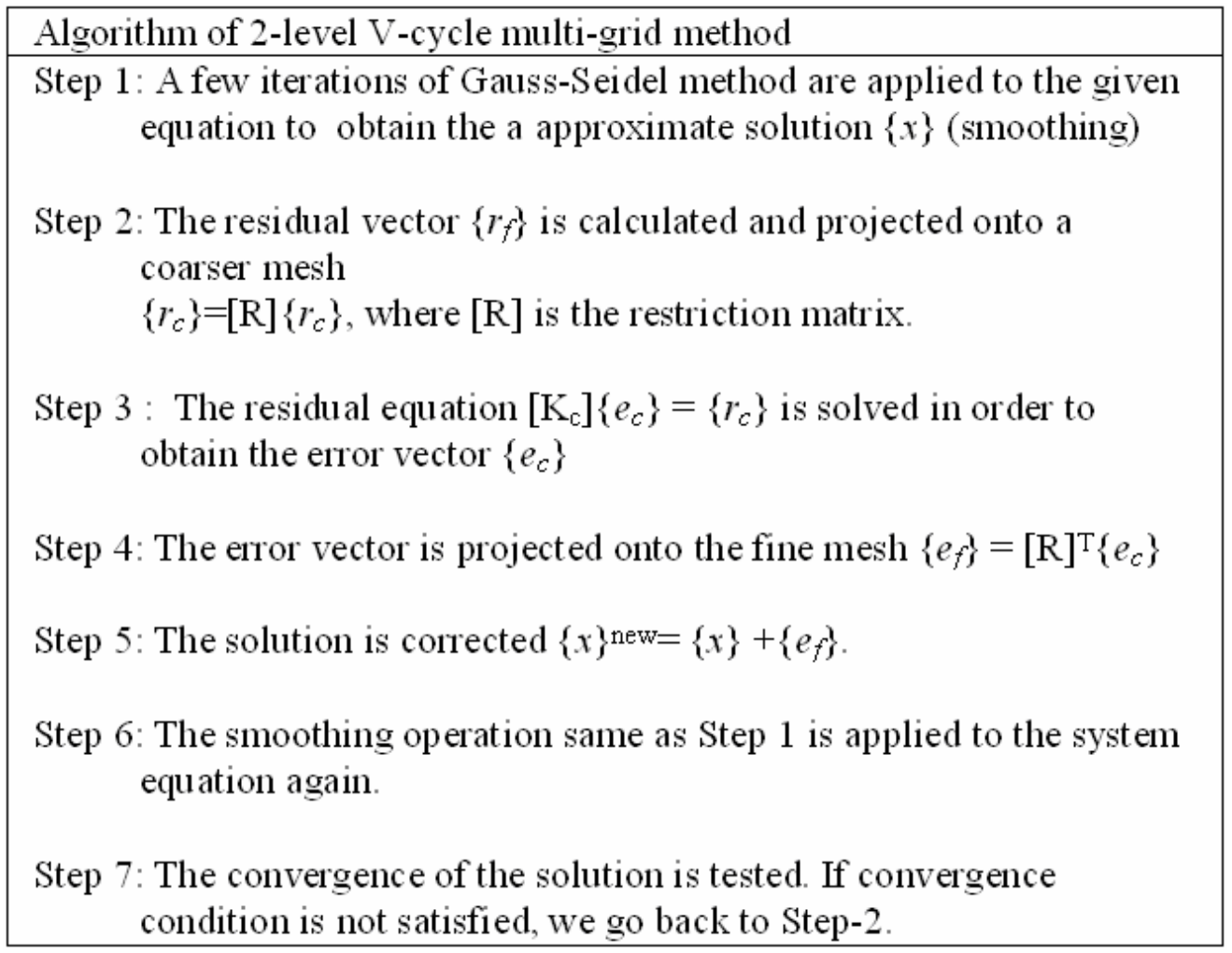




\section{Optimization of Perpendicular Magnetic Recording Head}

In this section, we analyze the design of a perpendicular magnetic recording head for hard disk drives. The model for this device is shown in Figure 4, and the objective of this problem is to maximize the flux density in the region $\mathbf{1}$ (recording flux), and to minimize the flux density in the region $\mathbf{2}$ (leakage flux affecting the adjacent bit). The permeability of magnetic material used is $1000 \mu_{0}$. This model is based on the 2-D model used by Okamoto (2005).
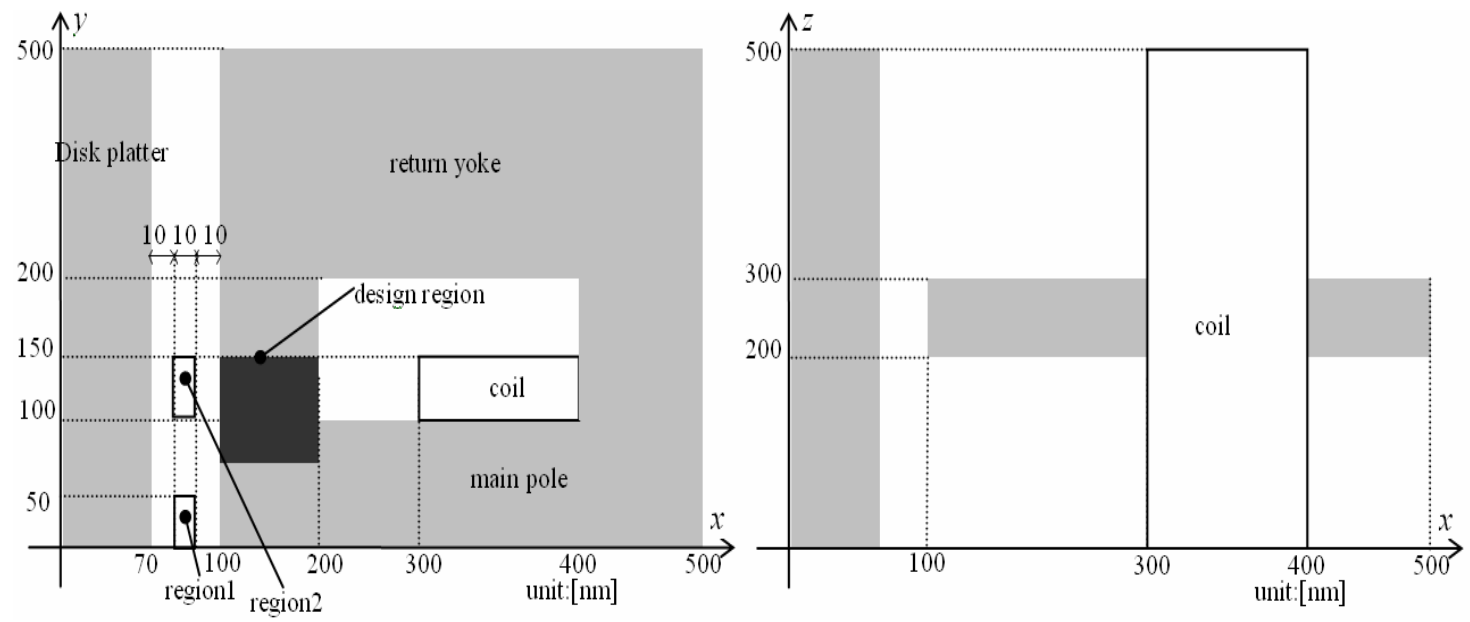

Figure 4: Analysis region for magnetic head. Current density in coil: $0.1 \mathrm{~A} / \mathrm{m}^{2}$; permeability of the magnetic material: $1000 \mu_{0}$.

The objective function, for a maximization problem, is defined as:

$$
f=-\left\{w_{1} \sum_{u=1}^{n_{1}} \frac{1}{\left(B_{1 x}^{i}\right)^{2}}+w_{2} \sum_{i=1}^{n_{2}}\left(B_{2 x}^{i}\right)^{2}\right\}
$$

where $B_{1 x}^{i}$ and $B_{2 x}^{i}$ are the $\boldsymbol{x}$ components of magnetic flux density in regions $\mathbf{1}$ and $\mathbf{2}$, respectively, and $w_{1}$ and $w_{2}$ are the weighting coefficients, which are chosen as 1 and 20000, respectively. The number of sampling points used is $n_{l}=120$ and $n_{2}=200$. 
In this paper, a $10 \times 10 \times 5$ partition of the design region was used. The characteristics of the meshes used for the multigrid FEM analysis are shown in Table I.

Table I: Coarse and fine mesh characteristics

\begin{tabular}{l|cc} 
Method used & Multigrid & ICCG \\
\hline CPU time [s] & 15.849 & 62.658
\end{tabular}

Table II shows a comparison of the average processing time required for the multigrid method and the conventional ICCG solver to evaluate the numerical model of the magnetic recording head (machine used: Pentium 4, 3.2GHz, 2GB RAM, Linux; computational time averaged over 1000 evaluations).

Table II: Calculation times for the multigrid method and the ICCG method in the magnetic recording head problem

\begin{tabular}{l|cc} 
& Elements & Unknowns \\
\hline Coarse Mesh & 4,840 & 16,377 \\
Fine Mesh & 38,720 & 123,482
\end{tabular}

It is clear from this result that the multigrid method outperforms the ICCG, greatly reducing the computational time required for the evaluation of the model. This feature makes the multigrid solver more interesting for coupling with the TopCSA for the solution of electromagnetic problems. 


\section{Optimization Results}

The TopCSA was applied for solving the problem described in the previous section. The algorithm parameters used were:

- $\quad N_{P o p}=4$

- $\quad N_{S e l}=0.7$

- $A_{s}=1.1$

- $\quad$ Ratio between Affinity Maturation and Global Operators = 7:3;

- Stop criterion: number of function evaluations $=5,000$.

Figure 5 shows the solution obtained. This solution found presents a value of $f=-385.175$. The terms of the objective function equation (2) are $\sum 1 /\left(B_{1 x}^{i}\right)^{2}=202.742$ and $\sum\left(B_{2 x}^{i}\right)^{2}=9.12165 \times 10^{-3}$ respectively, with average values of $1.689 T^{2}$ and $45.60825 \mu T^{2}$, respectively. By these criteria, the TopCSA was successful in finding a good optimum for the problem. Moreover, this result was found within a reduced computational budget.

\section{Conclusions}

In this paper, we have introduced a $3 \mathrm{D}$ formulation for a topology optimization algorithm. The TopCSA is an evolutionary approach that combines local exploitation and global exploration of the search space as a way to accelerate the convergence on computationally expensive problems, commonly found in many areas of engineering where the evaluation of numerical models is required.

The proposed approach was applied to the design of a perpendicular magnetic recording head, such as the ones found in magnetic hard disks. The solution found for the 

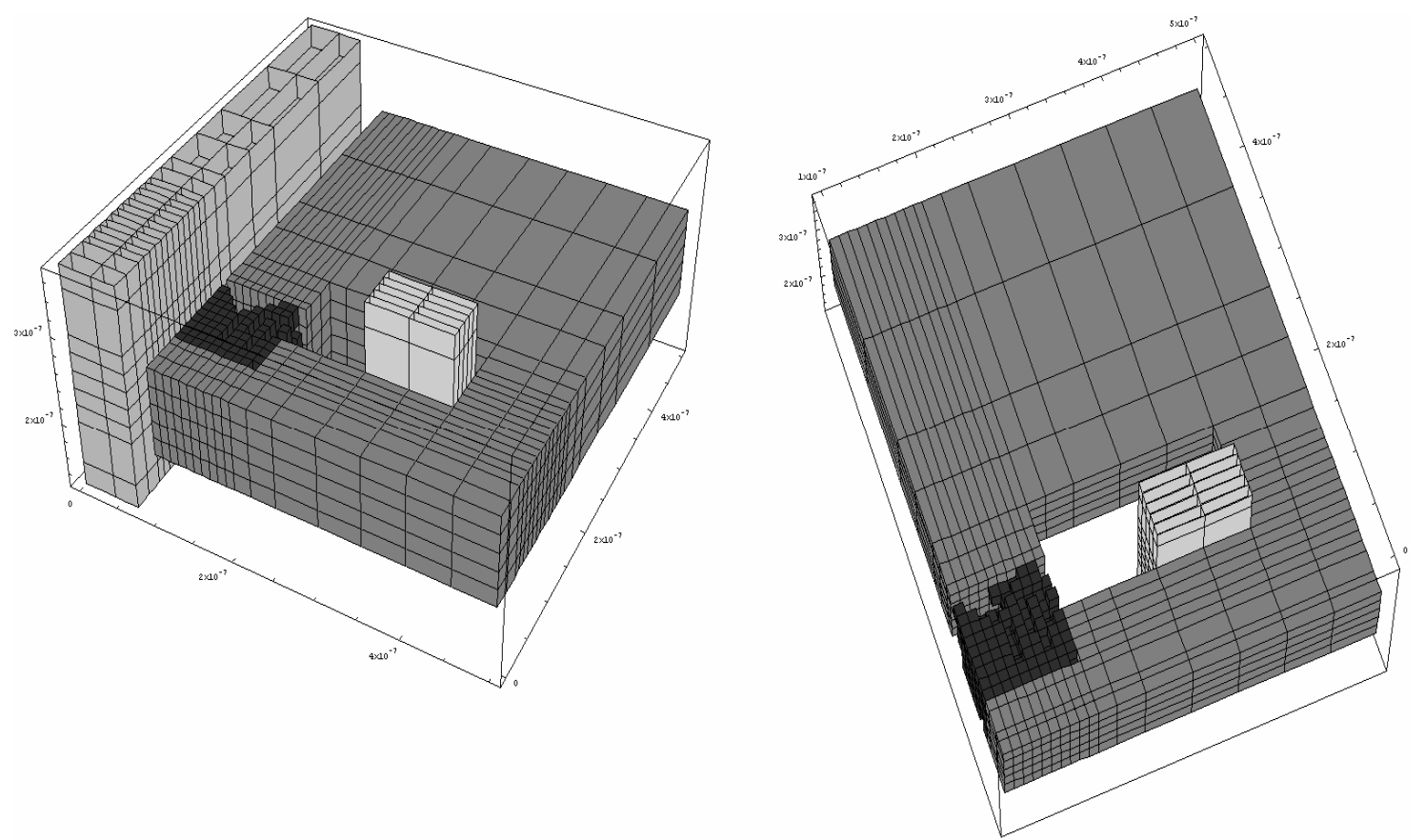

Figure 5: Two views of the result obtained by the TopCSA for the perpendicular magnetic recording head

problem was able to satisfy the design requirements, that is, the maximization of the magnetic field in a certain region of the space while keeping a low value in the neighboring region.

The development of better ways to generate smoother solutions, more interesting from an implementation point of view, is a natural next step on this research. Also, the use of the TopCSA for the solution of multiobjective problems, or of problems where more than two materials are involved, are possibilities to be explored in the future.

\section{Bibliography}

Bendsøe, M.P. (1988) "Generating optimal topologies in structural design using a homogenization method", Computational Methods in Applied Mechanical Engineering, Vol. 71, pp. 197-224. 
Bendsøe, M.P. (1999) "Material interpolation schemes in topology optimization", Archive of Applied Mechanics, Vol. 69, pp. 635-654.

Byun, J-K. (1999) "Topology optimization of electrical devices using mutual energy and sensitivity", IEEE Transactions on Magnetics, Vol. 36, No. 4, pp. 1144-1147.

Byun, J-K. (2004) "Node-Based Distribution of Material Properties for Topology Optimization of Electromagnetic Devices", IEEE Transactions on Magnetics, Vol. 40, No. 2, pp. 1212-1215.

Campelo, F. (2006) "An Immune-based Algorithm for Topology Optimization", Proceedings of the IEEE World Congress on Computational Intelligence (CDROM), Vancouver, Canada, July 16-21.

Chapman, C. (1993) "Genetic algorithms as an approach to configuration and topology design" Advances in Design Automation, Vol. 65, pp. 485-498.

De Castro, L.N. (2002) “Artificial Immune Systems: a New Computational Intelligence Approach", Springer-Verlag, London.

Dick, D. (1996) "Automated design of magnetic devices by optimizing material distribution", IEEE Transactions on Magnetics, vol. 32, no. 3, pp. 1188-1193.

Hamda, H. (2000) “Adaptive techniques for evolutionary topological optimum design”, in I. Parmee (ed.): Evolutionary Design and Manufacture, pp. 123-136.

Hamda, H. (2002) “Compact unstructured representations in evolutionary topological optimum design", Applied Intelligence, Vol.16, pp.139-155.

Icking, C. (2001) "VoroGlide version 2.2”, [Online Java Applet], http://www.pi6.fernunihagen.de/GeomLab/VoroGlide/ 
Im, C-H. (2003) "Hybrid genetic algorithm for electromagnetic topology optimization", IEEE Transactions on Magnetics, Vol. 39, No. 5, pp. 2163-2169.

Jakiela, M.J. (2000) "Continuum structural topology design with genetic algorithms", Computational Methods in Applied Mechanical Engineering, Vol. 186, pp. 339-356.

Kane, C. (1996) "Topological optimum design using genetic algorithms", Control and Cybernetics, Vol. 25, No. 5, pp. 1-25.

Mlejnek, H.P. (1993) “An engineer's approach to optimal material distribution and shape finding", Computational Methods in Applied Mechanical Engineering, Vol. 106, pp. 1-26. Nishiwaki, S. (1998) "Topology optimization of compliant mechanisms using the homogenization method", International Journal for Numerical Methods in Engineering, Vol. 42, pp. 535-559.

Okamoto, Y. (2005) "Magnetic shield design of perpendicular magnetic recording head by using topology optimization technique", IEEE Transactions on Magnetics, Vol. 41, No. 3, pp. 1788-1791.

Okamoto, Y. (2006) “3-D topology optimization of single-pole-type head by using design sensitivity analysis", IEEE Transactions on Magnetics, Vol. 42, No. 4, pp. 1087-1090. Rozvany, G.I.N. (1992) "Generalized shape optimization without homogenization", Structural Optimization, Vol. 4, pp. 250-252.

Schoenauer, M. (1996) "Shape Representations and Evolution Schemes”, Proceedings of the 5th Annual Conference on Evolutionary Programming, Cambridge, Mass., USA, pp. 121-129. 
Silva, E.C.N. (2000) "Topology optimization design of flextensional actuators", IEEE Transactions on Ultrasonics, Ferroelectrics, and Frequency Control, Vol. 47, No. 3, pp. $657-671$.

Wang, S. (2002) "Topology optimization of nonlinear magnetostatics", IEEE Transactions on Magnetics, Vol. 38, No. 2, pp. 1029-1032.

Watanabe, K. (2003) "Convergence of Multigrid Method for Edge-based FEM, IEEE Transactions on Magnetics, Vol. 39, No. 3, pp. 1674-1676.

Watanabe, K. (2006) "Topology optimization based on immune algorithm and multi-grid method", Proceedings of the 12th Biennial IEEE Conference on Electromagnetic Field Computation (CEFC'06), Miami, FL, USA, April 30-May 3.

Weisstein, E.W. (2003) "von Neumann Neighborhood", [Online], http://mathworld.wolfram.com/vonNeumannNeighborhood.html Yoo, J. (2000), "Structural optimization in magnetic devices by the homogenization design method", IEEE Transactions on Magnetics, Vol. 36, No. 3, pp.574-580.

Yoo, J. (2002), “Topology optimization for reduction of vibration caused by magnetic harmonic excitation", IEEE Transactions on Magnetics, Vol. 38, No. 6, pp.3643-3649. Yoo, J. (2004) "Modified Method of Topology Optimization in Magnetic Fields", IEEE Transactions on Magnetics, Vol. 40, No. 4, pp. 1796-1802. 